\title{
Hydroacoustic field in sonar cavity under mechanical excitations
}

\author{
Weike Wang ${ }^{1}$, Rui HUO ${ }^{2,3}$, Zhidong Wang ${ }^{2,3}$ \\ ${ }^{1}$ Key Laboratory of High Efficiency and Clean Mechanical Manufacture (Shandong \\ University) Ministry of Education, Ji’ nan 250061, China \\ ${ }^{2}$ School of Mechanical Engineering, Shandong University, Ji' nan250061, China \\ ${ }^{3}$ Key Laboratory of High Efficiency and Clean Mechanical Manufacture (Shandong \\ University) Ministry of Education, Ji' nan 250061, China

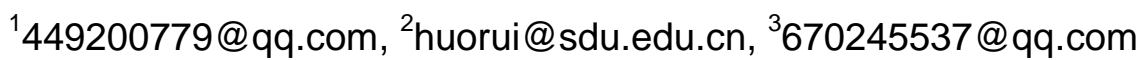

\begin{abstract}
Keywords: Sonar cavity, mechanical self-noise, fluid-structure interaction, hydroacoustic field.
Abstract. In view of the destructive influence of mechanical self-noise on sonar' $s$ detection capability at lower frequencies, a parametric model composed of panels and a water filled rectangular cavity is established for investigation of characteristics of hydroacoustic field caused by mechanical excitation loaded on sonar platform area. The analytical derivation and numerical simulation analysis of differential vibration equations provide theoretical reference for prediction and control of mechanical self-noise.
\end{abstract}

\section{Introduction}

The self-noise in sonar refers to the sonar sound interference at work, according to its source is divided into hydrodynamic noise, machinery noise, propeller noise and the ocean ambient noise. At present, many the self-noise in sonar problem discussions are in the majority with hydrodynamic noise, however, the mechanical noise problem also can' $t$ be ignored and particularly important for the low-speed submarine underwater navigation.Musha et al. Literature[1] proposed a method to determine the self-noise of sonar caused by turbulence by using a simplified acoustic vibration transfer function. Literature[2,3,4] based on the rectangular cavity model used the statistical energy method and the modal method to calculate and analyze the hydrodynamic component of sonar self-noise; In the literature[5], the low frequency $(10 \mathrm{~Hz} \sim 500 \mathrm{~Hz})$ self-noise of the platform area is predicted by using the finite element modeling of sonar platform area structure and empirical radiation coefficient.

In this paper, we first establish a parametric model of the noise analysis of rectangular cavity for sonar array mechanical cavity. Qualitative theoretical analysis is made to the modal frequency and influencing factors of fluid-structure interaction vibration and the influencing effect of acoustic window, providing a theory basis to the denoising of sonar array.

\section{Rectangular sonar array cavity model}

Model parameter definition

Fig. 1 rectangle filling water chamber on the panel is subject to external excitation pe elastic plate used to simulate sonar platform area and the rest of the panels are considered as rigid, the shadow part of the graph to simulates the acoustic window. Establishing in the Cartesian coordinate system OXYZ. The establishment of fluid solid coupling equation cavity

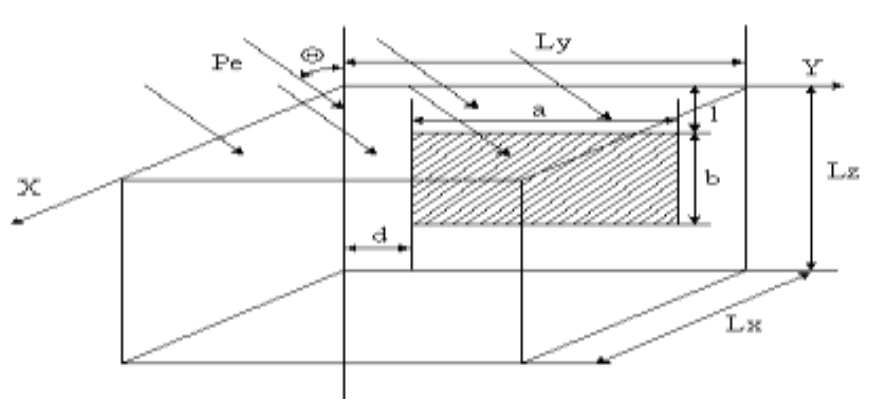

Fig. 1: An rectangular enclosure model for Sonar

Underwater acoustic field of water filling airtight chamber interior ministry satisfies wave equation 
$\nabla^{2} p(\sigma, t)-\left(1 / c_{0}^{2}\right) \partial^{2} p(\sigma, t) / \partial^{2} t=0$.

In this equation, $\mathrm{p}$ is the sound pressure, and $\mathrm{c} 0$ is the sound velocity.

If all the boundary are rigid, Eq.(1) has the general solution of the following form

$$
p=\rho_{0} c_{0}^{2} V \sum_{r} \frac{F_{r}(\sigma) \mathrm{e}^{\mathrm{j} \omega_{r}^{\mathrm{A}} t}}{\left(\omega_{r}^{\mathrm{A}}\right)^{2} M_{r}^{\mathrm{A}}}
$$

In this equation, $\mathrm{V}$ is chamber volume, $\omega_{r}{ }^{\mathrm{A}}, F_{r}{ }^{\mathrm{A}}$ and $M_{r}^{\mathrm{A}}$ are the each order natural frequencies, the natural vibration mode function and the modal mass of of the cavity interior underwater acoustic field in the rigid boundary conditions respectively.

When there is a non rigid boundary, referencing Gauss formula can be obtained ${ }^{[6]}$

$$
\ddot{P}_{r}+\left(\omega_{r}^{\mathrm{A}}\right)^{2} P_{r}=-A_{r} / V \quad(r=0,1,2, \ldots) \text {. }
$$

In this equation,

$$
\begin{aligned}
& P_{r}(t)=\frac{1}{V} \iiint_{V} \frac{p(\sigma, t) F_{r}(\sigma)}{\rho_{0} c_{0}^{2}} \mathrm{~d} v . \\
& A_{r}(t)=\oiint_{D} F_{r}(\sigma) a_{n}(\sigma, t) \mathrm{d} A .
\end{aligned}
$$

Consider the following that description of fluid solid coupling vibration with non rigid boundary. The modal equation obtained is

$$
M_{\mathrm{C} j}^{(t)}\left\{\ddot{q}_{j}^{(t)}+C^{(t)} \dot{q}_{j}^{(t)}+\left[\omega_{\mathrm{C} j}^{(t)}\right]^{2} q_{j}^{(t)}\right\}=Q_{\mathrm{C} j}^{(t)}+Q_{\mathrm{E} j}^{(t)} .
$$

$\omega_{\mathrm{C} j}{ }^{(l)}$ and $M_{\mathrm{C} j}{ }^{(l)}$ are respectively the natural frequency and modal mass of boundary structure $D_{\mathrm{C}}{ }^{(t)} . C^{(l)}=Z_{\mathrm{O}} / m_{\mathrm{C}}{ }^{(l)}, Q_{C j}{ }^{(t)}$ and $Q_{E j}{ }^{(t)}$ are respectively j order modal generalized incentives.

In the Eq. (5), let $a_{n}(\sigma, t)=\partial^{2} w^{(i)}(\sigma, t) / \partial t^{2}$, and substituting into Eq. (3), At the same time to express $p_{\mathrm{C}}{ }^{(l)}$ of Eq. (6), finishing available

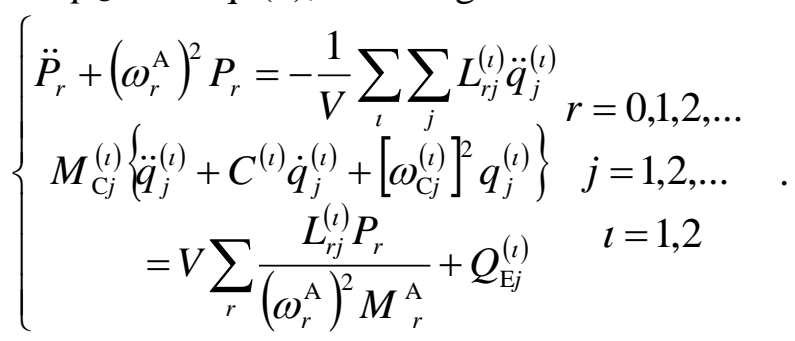

In this equation, $L_{r j}^{(\ell)}=\iint_{D_{\mathrm{C}}^{(t)}} F_{r}(\sigma) \Psi_{j}^{(\ell)}(\sigma) \mathrm{d} A$.

Eq. (7) which describes the water filled closed chamber in fluid and elastic cavity wall occurred flow solid coupling vibration differential equation (Group).

\section{Numerical simulation and analysis}

Modal frequency and influencing factors of fluid solid coupling vibration

Table 1 compares the acoustic modal frequency of rigid boundary rectangular cavity $f_{i}^{\mathrm{A}}=\omega_{i}{ }^{\mathrm{A}} / 2 \pi$, the modal frequency of elastic platform area wall board $D_{\mathrm{C}}{ }^{(1)} f_{\mathrm{C} i}{ }^{(1)}=\omega_{\mathrm{C} i}{ }^{(1)} / 2 \pi$ and the modal frequency $f_{i}$ of fluid-solid coupling vibration in two parts. $L_{X}=0.4 \mathrm{~m}, L_{Y}=0.6 \mathrm{~m}, L_{Z}=0.7 \mathrm{~m}, h^{(1)}=5 \times 10^{-3} \mathrm{~m}$, the material of elastic wall panel $D_{\mathrm{C}}{ }^{(1)}$ is the No. 45 steel.

Visible from Table 1, The first two order natural frequency (non-zero) of cavity fluid and elastic wall cavity coupling vibration is close to the natural frequency of elastic chamber wall. However, with the increase of the modal order, the modal frequency of the coupled vibration tends to be lower than that of the cavity wall structure of the corresponding order. Further analysis also shows that strengthening elastic wall rigidity can make high order of fluid-solid coupling vibration modal frequency increased more significantly, but it has little effect on the first few order of fluid-solid 
coupling modal frequency (Fig. 2); The change of fluid acoustic mode frequency in the cavity has little effect on the fluid-solid coupling modal frequency (Fig. 3).

\begin{tabular}{c|c|c|c}
\hline Order & $f_{i}^{\mathrm{A}}(\mathrm{Hz})$ & $f_{\mathrm{C} i}^{(1)}(\mathrm{Hz})$ & $f_{i}(\mathrm{~Hz})$ \\
\hline 0 & 0 & & $4.63 \times 10^{-5}$ \\
1 & 1071.43 & 107.963 & 109.2842 \\
2 & 1250 & 207.622 & 205.3723 \\
3 & 1646.35 & 332.195 & 247.3392 \\
4 & 1875 & 373.719 & 293.087 \\
5 & 2142.86 & 431.854 & 460.0024 \\
6 & 2159.53 & 597.951 & 467.6415 \\
7 & 2253.47 & 606.256 & 490.819 \\
8 & 2480.79 & 705.915 & 578.766 \\
9 & 2495.21 & 805.573 & 643.0509 \\
10 & 2500 & 830.488 & \\
\hline
\end{tabular}

Table 1: various modal frequencies of the rectangular cavity model

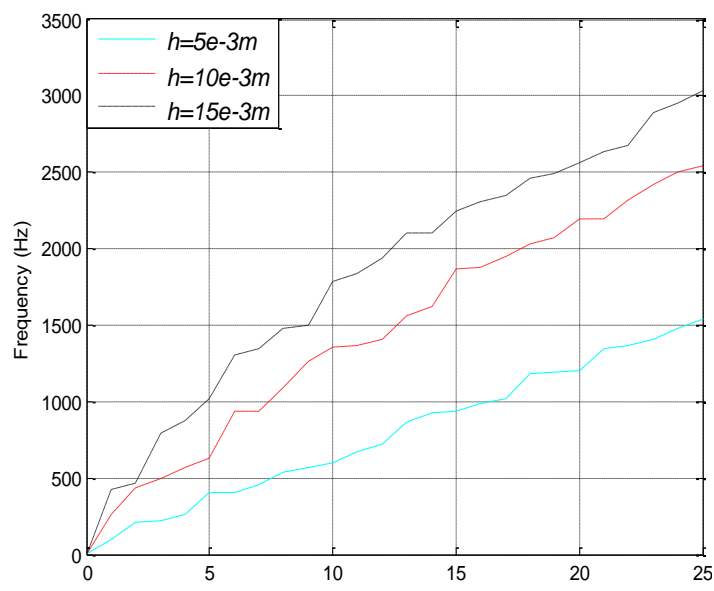

Fig. 2: Influence of $D_{\mathrm{C}}^{(1)}$, s thicknesson coupling modal frequencies

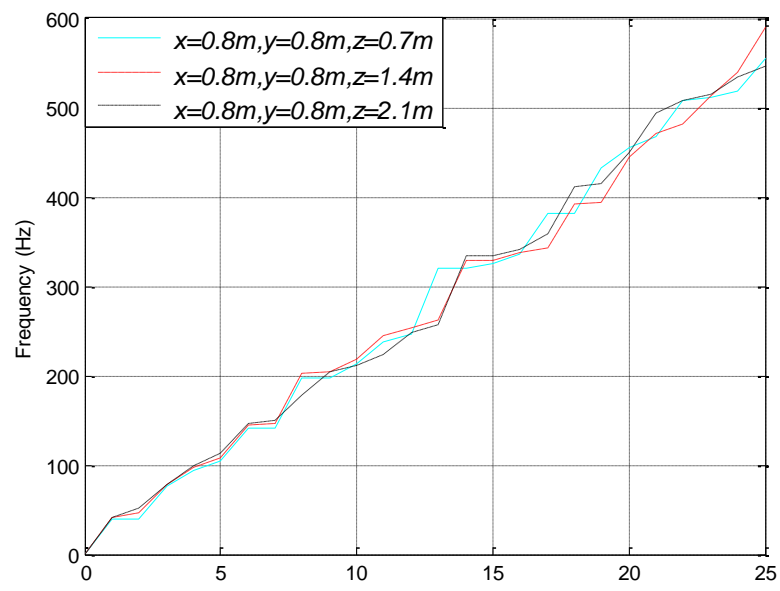

Fig. 3: Influence of cavity hight $L_{Z}$ on coupling modal frequencies

The influence effect of acoustic window

The influence of acoustic window has not been considered in calculation in a lot of literatures, the acoustic window in the actual system usually adopts glass steel or titanium alloy material, and making it have approximately the same acoustic impedance ratio with the outside sea water, so acoustic window can also be approximately regarded as absorbing boundary.

Fig. 4 is introduced through the different acoustic pressure spectrum of cavity acoustic window boundary, it is visible that the acoustic window boundary has little influence on the vibration modal, but reduces the acoustic pressure peak of resonance frequency. In addition, the hydroacoustic pressure at each point in parallel with the sound source in the plane is roughly the same. And the hydroacoustic pressures at different depth in the lower frequency are roughly equal, but in the medium-high frequency domain, different sound source distances make larger difference with spectrum of hydroacoustic pressure.

Fig. 5 shows that the different sizes of the acoustic window border are not affected by the coupling mode frequency of the cavity. The large acoustic window can reduce the sound pressure resonance peak, but also reduces the noise attenuation in the anti resonance frequency band. 


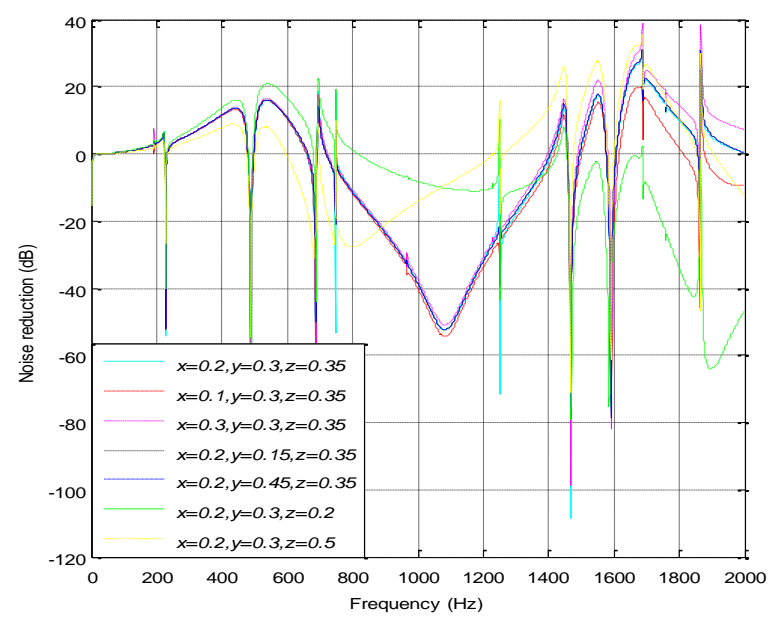

Fig.4: Hydroacoustic pressure under consideration of sonar window

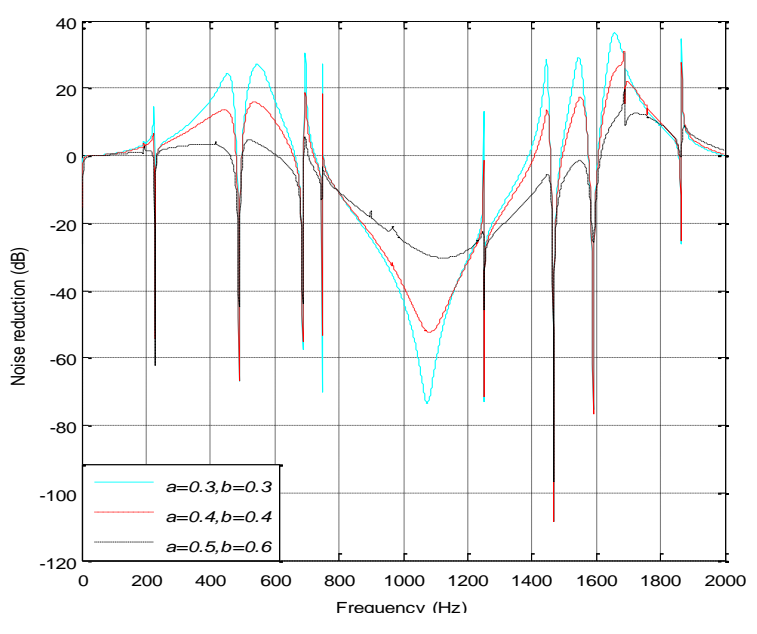

Fig. 5: Hydroacoustic pressurewith different sonar window size

\section{Conclusion}

The model of fluid-solid coupling vibration of plate and rectangular cavity with filling water was established and the underwater acoustic field was solved in this paper. The mechanical self-noise in sonar array cavity was qualitatively analyzed from theory.

The low order natural frequency of coupling vibration between cavity fluid and elastic chamber walls is more related to the structure modal frequency of elastic chamber wall than acoustic modal frequency of cavity fluid.

Acoustic window had a main influence on water acoustic field in the cavity that it could reduce the peak values of sound pressure at the resonant frequency and noise attenuation at the anti-resonant frequencies.

From the view of optimal design, the structure shape and size of cavity had great effect on its internal acoustic characteristics of underwater acoustic field, the influencing rule was very complex. With the increase of rigidity of the elastic wall plate, the number of the excited modal could be reduced; but it had little effect on the basic features of frequency distribution of underwater acoustic field.the Text

\section{References}

[1] Musha T, Kikuchi T. Numerical calculation for determining sonar self noise sources due to structural vibration[J]. Applied Acoustics. 1999, 58(1): 19-32.

[2] Mengsa Yu, Dongsheng Li. Calculation of hydrodynamic component of sonar self-noise by SEA method[J]. Journal of ship mechanics, 2004, 8(1): 99 105.

[3] Mengsa Yu, Zhu Zhengdao. Calculation of hydrodynamic component of sonar self-noise by integro-SEA method[J]. Journal of ship mechanics, 2007, 11(2): 273 283.

[4] Xiaobin Liu, Shijin LÜ, Mengsa Yu. Prediction and test of cavity' s hydrodynamic self noise induced by turbulent boundary layer[J]. Acta Acustica, 2015, 40(6): 845 849.

[5] Hualin Guo, Dongping Luo, Meixia Chen, etc. Prediction method for low frequency of self-noise in submarine' s forebody sonar platform area[J]. Ship science and technology. 2006, 27(4): 74 77.

[6] Dowell E H, Corman G F, Smith D A. Acoustoelasticity: general theory, acoustic natural modes and forced response to sinusoidal excitation, including comparisons with experiment[J]. Journal of sound and vibration, 1977, 52(4): 519 542. 